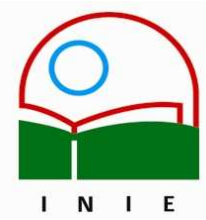

Actualidades Investigativas en Educación

Revista Electrónica publicada por el

Instituto de Investigación en Educación

Universidad de Costa Rica

ISSN 1409-4703

http://revista.inie.ucr.ac.cr

COSTA RICA

\title{
REFLEXIONES PARA CONSOLIDAR LA ÉTICA INTERCULTURAL A TRAVÉS DE LA EDUCACIÓN EN VALORES Y LA EDUCACIÓN INTERCULTURAL
}

MEDITATE TO CONSOLIDATE THE INTERCULTURAL ETHICS TO INCLINATION OF THE INTERCULTURAL EDUCATION

Volumen 8, Número 2

pp. $1-18$

Este número se publicó el 30 de agosto 2008

Egilde Zambrano van Beverhoudt

Ana Prieto Sánchez

La revista está indexada en los directorios:

LATINDEX, REDALYC, IRESIE, CLASE, DIALNET, DOAJ, E-REVIST@S,

La revista está incluida en los sitios:

REDIE, RINACE, OEI, MAESTROTECA, HUASCARAN

Los contenidos de este artículo están bajo una licencia Creative Commons 


\title{
REFLEXIONES PARA CONSOLIDAR LA ÉTICA INTERCULTURAL A TRAVÉS DE LA EDUCACIÓN INTERCULTURAL
}

\author{
MEDITATE TO CONSOLIDATE THE INTERCULTURAL ETHICS TO INCLINATION OF THE \\ INTERCULTURAL EDUCATION
}

\section{Egilde Zambrano van Beverhoudt ${ }^{1}$ Ana Prieto Sánchez ${ }^{2}$}

\begin{abstract}
Resumen: El propósito de este documento es reflexionar sobre una propuesta de consolidación de la ética intercultural a partir de la educación intercultural. Se efectuó un análisis documental basado en autores con pertinencia en el área de interés. Para el desarrollo teórico se analizaron aspectos como globalización, educación, valores, multiculturalidad e interculturalidad. Se concluye que la ética intercultural minimiza los riesgos de la globalización y busca la construcción de sistemas de valores que aseguren la convivencia social. La educación debe asumir su papel activo en la consolidación de la ética intercultural. La interculturalidad es un modelo de gestión que administra con eficiencia y eficacia la diversidad cultural.
\end{abstract}

Palabras clave: ÉTICA INTERCULTURAL, EDUCACIÓN EN VALORES, EDUCACIÓN INTERCULTURAL, GLOBALIZACIÓN, VALORES

\begin{abstract}
The purpose of this document is to meditate on a proposal of consolidation of the ethical intercultural starting from the education intercultural. A documental analysis was made based on authors with relevancy in the area of interest. For the theoretical development aspects like globalization, education, values, multiculturaly and interculturaly were analyzed. It is concluded that the ethical intercultural minimizes the risks of the globalization and search the construction of systems of values that they assure the social coexistence. The education should assume its active paper in the consolidation of the ethical intercultural. The interculturaly is an administration model that administers with efficiency and effectiveness the cultural diversity
\end{abstract}

Keywords: INTERCULTURAL ETHICS, EDUCATION IN VALUES, INTERCULTURAL EDUCATION, GLOBALIZATION, VALUES

\footnotetext{
1 Doctora en Ciencias Humanas de la Universidad del Zulia. Profesora Asociada de LUZ COL. Adscrita al Programa de Promoción al Investigador (PPI). Universidad del Zulia (LUZ). Núcleo Costa Oriental del Lago (COL). Cabimas, Venezuela. Dirección electrónica: egilde@yahoo.es

2 Doctora en Ciencias Humanas de la Universidad del Zulia. Profesora Asociada de LUZ COL. Adscrita al Programa de Promoción al Investigador (PPI). Universidad del Zulia (LUZ). Núcleo Costa Oriental del Lago (COL). Cabimas, Venezuela. Dirección electrónica: anaprieto@cantv.net
}

Artículo recibido: 25 de febrero, 2008

Aprobado: 6 de agosto, 2008 


\section{Introducción}

Hoy en día, cuando el mundo está inserto dentro del proceso globalizador, presenciamos la ruptura de fronteras entre países en procura de su alineación, primero económica, más luego, con una similar tendencia en lo político y social. Frente a esta tesis homogeneizadora, surgen fuertes luchas por conservar las identidades y así, las sociedades se van reconstruyendo en la diversidad, es decir, en la sumatoria de diferentes culturas y subculturas; estas últimas se constituyen por grupos de personas con comportamientos que las diferencian de la cultura de la cual forman parte. En una cultura pueden habitar subculturas que crecen a expensas de ella, pero que desarrollan creencias, costumbres y estilos de vida distintos. Esta dinámica caracteriza aún mas la heterogeneidad de las sociedades.

En este panorama diverso, existe el riesgo de que los seres humanos simplemente se limiten a coexistir los unos con los otros, comprometiéndose seriamente el porvenir de la humanidad.

Así las cosas, en este ensayo se aborda la ética intercultural como un planteamiento que apunta a la posibilidad de que exista un futuro para los habitantes del planeta. Para que esta posibilidad sea cierta, los seres humanos deberán comprometerse consigo mismos y con su entorno, a fin de garantizar, no ya la supervivencia, sino la convivencia.

La ética intercultural nos advierte sobre los peligros de no asumir el juego del intercambio cultural (con todo lo que significa) al que nos lleva la globalización, para así, sobre el reconocimiento de las conflictividades, "reproducir la vida humana comunitaria y personal" (Salas, 2002, p.24). Es en este contexto globalizado en donde la ética intercultural se justifica al reconocer la existencia de la diversidad cultural y poner de cara las diferencias, proponiendo el diálogo para el alcance de la convivencia, reconociendo la existencia del otro. Y es que la ética intercultural propone construir un espacio donde, en la diversidad, pueda lograrse el entendimiento social a través de la inclusión.

En la intención de que la ética intercultural logre su consolidación como una metodología de vida que garantice las relaciones positivas y enriquecidas entre los miembros de las sociedades diversas, se propone en este documento que la ética intercultural se apoye en una educación intercultural, la cual contempla a su vez una educación en valores. Estos dos 
pilares permitirán enfrentar los riesgos que entraña el proceso de la globalización, permitiendo alcanzar la convivencia social armónica.

\section{La ética intercultural en el contexto de la globalización}

Mucho se ha dicho acerca de la globalización y muchos desacuerdos han surgido en la discusión sobre el tema, pero el punto de encuentro entre todas las tendencias del pensamiento es que, queramos o no, la globalización acerca de alguna manera a todas las personas. "Podemos ser ciudadanos de un país, miembros de una comunidad específica y creyentes de una religión particular, pero cada vez coparticipamos más en una economía única, entretejida e interdependiente"(Dalla, 1999, p.13).

Sin embargo, de esta realidad económica indetenible surge una realidad humana mucho más profunda, y es que el proceso de la globalización pone de manifiesto las desigualdades entre los países, entre los individuos, entre sus culturas, y entre sus intereses, beneficiando a algunos y perjudicando a otros.

Según Fornet-Betancourt (2001), el fenómeno de la globalización nos confronta hoy con un proceso de consecuencias aplastantes que cambian y transforman nuestras condiciones de vida. En lo esencial, la globalización es actualmente el resultado de una expansión incontrolada del neoliberalismo político y económico con la finalidad de homogeneizar y monotonizar el planeta según las exigencias del mercado capitalista.

Por su parte, Dalla (1999) expresa que ciertamente la globalización nos ha transformado en una aldea global, pero no en una aldea homogénea, por lo que las diferencias entre culturas, países, regiones y vecinos parecen más agudas y cada vez más fundamentales; se hace imperativo resolver la tensión entre un solo mundo y su multiplicidad de partes.

Huntington (1996, citado por Dalla, 1999, p. 34) afirma que "el mundo se está fracturando cada vez más, con culturas y civilizaciones que se rechazan entre sí con el propósito de retener su distinción ante la globalización despersonalizadora”.

Mucho se ha discutido sobre la crisis de valores que atraviesan las sociedades, toda una historia de conflictividad fundamentada en la dialéctica de la negación del otro (Salas, 2002), el desarraigo de valores tradicionales, así como la proliferación de otros negativamente 
contextualizados, ha quebrantado los cimientos de la sociedad y propiciado el enfrentamiento entre sus individuos. Aunado a esto, la existencia de la diversidad cultural, aun dentro de los mismos grupos sociales, ha originado que la convivencia sea, en ocasiones, un ideal difícil de alcanzar.

Al ser entonces la globalización una realidad de la que no se puede escapar, ya que se quiera o no, se está inmerso en ella, se hace necesario pensar en cómo posibilitar la convivencia frente a los riesgos que supone. La respuesta está en el reconocimiento de una ética intercultural que promueva el respeto a la vida, a las diferencias y al diálogo.

Hoyos (1998) justifica la ética intercultural al plantear la necesidad de fortalecer la formación en la competencia comunicativa a objeto de permitir la apertura de espacios globales para razonar y encontrar el diálogo y la convivencia que permitan enfrentar la intolerancia y exclusión propias de este tiempo. Al respecto, la Asociación de Enseñantes con Gitanos (1996) señala que la perspectiva intercultural debe suponer un instrumento fundamental en la lucha contra la injusticia social.

La ética intercultural no es solo un mero desarrollo teórico-filosófico, es una propuesta de acción que exige el compromiso de los individuos, quienes, conscientes de sus diferencias, buscan la construcción y reconstrucción de un sistema de valores en el cual todos tengan cabida y así asegurar la convivencia social; requiere de participación y responsabilidad. La ética intercultural apuesta por la búsqueda y el encuentro del entendimiento en condiciones de diversidad, lo cual ciertamente constituye un desafío; sin embargo, esto es posible cuando hombres y mujeres concientizan su papel activo dentro de las sociedades en las cuales interactúan y se reconocen mutuamente, desde y a pesar de sus diferencias.

\section{3. Ética intercultural y educación}

Puede afirmarse que en el mundo globalizado, el ser humano y los pueblos pueden ser objeto de múltiples formas de dominación; pero tienen también la posibilidad de construir y reformular, a través de la educación, un espacio más propicio para el crecimiento de una verdadera solidaridad humana. Tal relación, globalización-condición humana, quedará determinada por la educación de una ética global, capaz de sistematizar significados y valores sobre los que se construirán las relaciones entre los pueblos, las organizaciones, las 
personas y de todos con la naturaleza (Toro, 1998; Guedez, 2001, citados por Ferrer y Romero, 2003)

Ciertamente, la contribución de la educación en la construcción e implementación de la ética intercultural es fundamental; esto puede verse plasmado en el siguiente párrafo:

La educación debe preparar su terreno. Reducida hoy, con demasiada frecuencia, a la distribución superficial del saber y a la consolidación de las divisiones sociales o de los valores de un mundo agonizante, debe romper con sus cuadros muertos para elaborar una formación del hombre total, ofrecida a todos por igual, dejando a cada uno libre frente a sus últimas perspectivas, pero preparado para la vida común de los hombres equilibrados, fraternalmente preparados los unos con los otros para el oficio del hombre (Kant, 1994, citado por Arrieta y Otros, 1995, p.1).

El autor hace un llamado para que la educación asuma su protagonismo en la formación de un ser humano que debe saber convivir.

En este sentido, la educación tiene el gran desafío de formar para el respeto al pluralismo, al consenso y a la convivencia en el marco de un contexto en crisis de valores, intolerancia, violencia e inequidad económica y social (Flimus, 1998), que enfatice en la formación de valores y en la lucha por una democracia que debe educar en los valores del respeto a todo individuo, por la igualdad de los derechos y la justicia social (Petty, 1998).

Bien lo expresa la Comisión Pedagógica de S.O.S. Racismo Madrid (1997) cuando afirma que la educación constituye un derecho básico de todo ser humano cuyo desarrollo efectivo ha de ser considerado con valor de axioma por cualquier sociedad que pretenda lograr un auténtico clima de respeto y convivencia a partir de la lucha contra toda forma de discriminación entre personas, facilitando la integración social de todos los individuos y la conservación de su igualdad de oportunidades, para corregir, de esta forma, las desigualdades que se derivan del desarrollo social.

Se habla entonces de una educación que valore la diversidad y que concientice a los actores de este mundo diverso a entenderse e integrarse en un proyecto común: la convivencia dentro de un mismo planeta. 
En una sociedad pluralista, la escuela debe generar el respeto, la valoración y la participación de todos los alumnos y las alumnas en términos de igualdad (Millán, 2001) pues, en principio, cumplir con esta función le permitirá enfrentar las demandas de una sociedad diversa. Los docentes y las docentes deben asumir el rol que les corresponde en el sentido de diseñar estrategias que les permitan responder con pertinencia a la diversidad cultural de sus aulas, lo que constituye una muestra representativa de la diversidad social, regional, nacional y mundial; dejar de lado la visión meramente cognoscitiva de la cátedra para profundizar en el desarrollo de la integralidad del educando; prepararlos para que sean capaces de actuar socialmente basados en la comprensión crítica de una realidad que es diversa.

Los educadores y las educadoras tienen la inmensa responsabilidad de educar en la interculturalidad; han de tener una visión amplia de la educación, identificando en ella los rasgos culturales, políticos y sociales para dejar de ser, junto con la escuela, los agentes reproductores de la ideología social del Estado y de la clase dominante (Millán, 2001).

Jordán y Otros (2002) explican que aunque el interculturalismo es una filosofía política, moral y educativa en fase de construcción, se caracteriza por la consideración de la sociedad como una realidad poliforme de grupos que tienen su propia subcultura (mujeres, ancianos, homosexuales, jóvenes, inválidos, desempleados y demás ciudadanos) que luchan por el reconocimiento de sus derechos, así como las minorías culturales (entendidas tradicionalmente) luchan por el reconocimiento de los suyos.

Los sistemas educativos deben entonces reconocer que existen dentro de sociedades poliformes, que deben atender sus necesidades y que la heterogeneidad es la realidad en la cual se desarrollan, por lo tanto deben asumirla.

La ética intercultural atiende la diversidad cultural de todas las sociedades desde los principios de igualdad, interacción y transformación social; implica una forma de entender y vivir las relaciones sociales. Se materializa a través de una manera de plantear y desarrollar el hecho educativo (Asociación de Enseñantes con Gitanos, 1996). Interculturalidad y educación van de la mano; la primera requiere de la segunda para hacerse escuchar, ser internalizada por los individuos y practicada con convicción. 


\section{Educar para la interculturalidad y educar en valores: fundamentos de una ética intercultural}

Martínez (2000, p.2), al referirse a la razón de ser del educar para la interculturalidad, expresa que ésta se preocupa por el "cómo podemos reconstruir las competencias humanas para vivir en paz; para hacer las paces". La educación para la interculturalidad debe estudiar las maneras que tienen los seres humanos de asumir responsabilidades para cultivar las relaciones entre sí mismos y la tierra. Es una educación para una ciudadanía sin fronteras. EI autor hace énfasis en que la educación para la interculturalidad es una posibilidad para lograr la convivencia, para entendernos entre diferentes, para educar en el respeto y la diversidad.

Así las cosas, la orientación intercultural se consolida en el compromiso por asegurar la convivencia entre los miembros de diferentes culturas y subculturas, pues tales diferencias son expresión de la riqueza de una sociedad en la búsqueda de respuestas a las necesidades de la vida (Jordán y otros, 2002).

Aceptar la realidad globalizadora implica poner los pies en la tierra y plantear salidas a las consecuencias que ésta genera. En un afán por unificar, pareciera que resaltan las diferencias; en la premisa de homogeneizar las relaciones entre los países algunos salen aventajados y otros sufren las desventajas, las desigualdades entre países ricos y pobres se tornan más evidentes y las imposiciones son la práctica común para permitir la supervivencia de los más débiles.

Retomando las ideas iniciales, es imprescindible buscar la metodología adecuada para hacer frente a este contexto, y es a través de la ética intercultural que lograrían plantearse relaciones positivas en medio de la diversidad en todos los planos, porque si bien existe una diversidad mundial no es menos cierto que dentro de un mismo país la diversidad es patrón de vida. Poner en práctica un proyecto de ética intercultural no tendría sentido si primero no se fundamenta en una educación para la interculturalidad: reconocer la diversidad cultural, sacar el mayor provecho de su riqueza a través de la educación y hacérselo entender a la gente.

Tal y como lo expresan Jordán y Otros (2002), la meta de la educación intercultural no es aprender la cultura del otro, sino aprender a partir del encuentro con el otro. Los defensores 
de la interculturalidad mantienen la consideración de las diferencias culturales como variaciones en la búsqueda de soluciones a los problemas humanos; por tanto, siempre es mucho más lo que une a las personas que lo que las separa, apareciendo una perspectiva universalista y la necesidad de afirmación de los valores comunes.

La integración cultural debe plantearse como el enriquecimiento mutuo de culturas mayoritarias y minoritarias como principio básico de la interculturalidad. Para ello es necesaria una verdadera educación intercultural capaz de acoger la diversidad, que modifique estereotipos y prejuicios, favorezca el conocimiento de las culturas minoritarias y promueva actitudes, conductas y cambios sociales que eviten la discriminación y favorezcan las relaciones positivas (Comisión Pedagógica de S.O.S. Racismo Madrid, 1997).

Es importante acotar, que si bien la educación intercultural es prioritaria en la operacionalización de una ética intercultural, ésta no podría llevarse a cabo sin tomar en cuenta la educación en valores.

Expresan Arana y Batista (2000, p. 4), que "La educación en valores es un proceso sistémico, pluridimensional, intencional e integrado que garantiza la formación y el desarrollo de la personalidad consciente; se concreta a través de lo curricular, extracurricular y en toda la vida universitaria." Su forma de organización la constituye el proyecto educativo.

Desde el punto de vista socio-educativo, los valores son considerados referentes, pautas o abstracciones que orientan el comportamiento humano hacia la transformación social y la realización de la persona. Son guías que orientan la conducta del ser humano y de cada grupo social (Denis, 2000). Los valores son el conjunto de creencias básicas, esqueleto o diseño arquitectónico que da sentido y coherencia a la conducta del ser humano (Ortega y Mínguez, 2001) y representan las normas ideales de comportamiento sobre las que descansa la cultura como un modo de vida integrado (Siliceo y otros, 1999).

Los valores se fijan por aprendizaje social desde nuestros primeros años de vida, principalmente por la interacción entre padres, madres, hijos e hijas y también por la influencia de la educación. Adicionalmente, Gómez y otros (2004) consideran que los valores pueden construírse a través del autoconocimiento, al análisis crítico y la toma de conciencia. El modelaje de personas con influencia también es fuente para la construcción de valores. 
Así, la importancia de educar en valores, de afianzar estas pautas en los individuos que les permitirán consolidar puntos de referencia para actuar correctamente, orientando su comportamiento hacia el alcance de metas. El proceso de educar en valores facilita los cimientos sobre los cuales se ha de construir la convivencia; "a la educación le toca la trascendental tarea de educar en valores a través de todas las actividades que le son propias ya que, todos los caminos deben llevar a ese destino" (Ramos, 2000, p. 55).

Educar en valores implica un proceso inagotable de formación donde se prepara al ser humano para la vida, una vida en sociedad fundamentada en valores como la disposición al diálogo, tolerancia, libertad, solidaridad, justicia y paz, pues son estos valores los que posibilitan la convivencia y que hoy día, cuando se habla de globalización e interculturalidad cobran mayor sentido y vigencia.

\section{Valores que fortalecen la ética intercultural}

La ética intercultural, en su práctica, se ve fortalecida a través de los valores que a continuación se presentan.

\section{El diálogo}

Está claro que el proceso globalizador ha derribado fronteras y al mismo tiempo ha intentado unificar los espacios. Sin embargo, partiendo de la intención inicial de homogeneizar, ha derivado que, en vez de lograr armonías, han destacado las diferencias. Estas diferencias, provenientes de identidades diversas, han generado que resalten los desacuerdos y los conflictos disfuncionales.

Se hace necesario entonces, acudir al diálogo (puesta en práctica de la verdadera comunicación) para acortar las distancias entre las personas y propiciar los acuerdos.

Expresan Ortega y Mínguez (2001, p. 40) que "quizás nunca como hoy en una sociedad desgarrada por tantos enfrentamientos, sacudida por fundamentalismos de todo tipo, se hace tan necesario el diálogo como procedimiento para la solución de nuestros conflictos". Luego el diálogo se construye en el entendimiento activo, cuando los individuos, de manera consciente, buscan solventar sus diferencias para compartir espacios sociales y lograr la convivencia. 
En la práctica del verdadero diálogo, las personas que intervienen deben disponerse a escucharse, a modificar sus posiciones ante argumentos convincentes, a buscar soluciones correctas a partir del acuerdo mutuo y a comprometerse a que la decisión tomada atienda a los intereses de todos los afectados (Gómez y Otros, 2004).

\section{La tolerancia}

La tolerancia implica el respeto y la defensa de la libre expresión de las ideas y modos de vida, independientemente de que no sean compartidos por todos, pues profundiza en la acogida de otro que es diferente (Ortega y Mínguez, 2001). Es un valor fundamental para garantizar la convivencia entre las personas, pues para convivir con la diversidad es necesario ser tolerante.

Expresa Díaz (2005, p. 2) que:

La tolerancia es una virtud encomiable. Quien es tolerante es una persona digna de respeto y admiración, puesto que es capaz de aceptar a los demás y de esta forma se destaca por su capacidad gregaria. Valoramos a quienes son capaces de salir de sí y de encontrarse con otro, aún a pesar de sus diferencias.

Afirma Ramos (2000) que en una educación global debe cultivarse el respeto a la diversidad. El respeto se operacionaliza a través de la tolerancia, es decir, del conocimiento y reconocimiento de la individualidad del otro.

Y es que la tolerancia permite la construcción de los espacios necesarios para que existan puntos de encuentro ante las diferencias personales, sociales, culturales, religiosas y de cualquier otra índole.

\section{La libertad}

La libertad es la posibilidad de que las personas sean ellas mismas, de que se puedan desarrollar (Ramos, 2000). Tener libertad es saberse poseedor de un derecho a disfrutar de espacios con libertad de expresión, de movimiento y de juicio, y es también el derecho a participar activamente en las decisiones sociales que involucren a las personas.

En un contexto globalizado, expresado en una dominación comunicativa que ahoga las expresiones culturales e ideológicas diversas (Ortega y Mínguez, 2001), se hace necesario el 
rescate de la libertad como valor que devela la autonomía de las personas en la defensa de lo que cada una significa, de sus ideales, de sus puntos de vista, de sus costumbres y de sus particularidades. La libertad debe configurarse como un estilo de vida que, alejado del mero discurso filosófico, se fundamenta en el valor de poder ejercerla: ser libre, sentirse libre y practicar esa libertad.

\section{La solidaridad}

Para Ramos (2001, p.45), "la solidaridad consiste en un sentimiento de comunidad, de afecto hacia el necesitado, de compartir no solo cosas, sino también obligaciones". La solidaridad entraña un sentido de responsabilidad hacia el otro que se traduce en una vinculación de las personas entre sí. Por ser social, el ser humano se desarrolla en comunidad, y en comunidad entiende que cada quien necesita de los demás. Esta necesidad se encarna en la solidaridad; en este sentido, los intereses de la comunidad prevalecerán sobre los individuales en la búsqueda del bien común.

Según García (1994), el concepto de solidaridad puede descomponerse en tres aspectos esenciales:

- Compasión. La solidaridad es un sentimiento que determina y orienta la forma de ver y acercarse a la realidad humana y social, condicionando su perspectiva y horizonte; supone un sentimiento fundamental de fraternidad.

- Reconocimiento. No toda compasión genera solidaridad, solo aquella que reconoce al otro en su dignidad como persona.

- Universalidad. La solidaridad trasciende todas las fronteras (políticas, religiosas, territoriales, culturales, etc.) y se instala en cualquier hombre y mujer.

Para Sarasola (2000, p.100), "la solidaridad es una respuesta a la injusticia, ya que en nuestra sociedad no todos participamos de la riqueza de la misma manera". Luego la solidaridad, como valor que guía el comportamiento del ser humano, le llevará a emprender acciones para contribuir con el bienestar de quienes le rodean, esto es, ayudar al que lo necesita. Es hacer algo por los demás.

\section{La justicia}

Afirma Siliceo (1997, p.37), que el reto de los líderes es crear un mundo mas justo, y al respecto señala: 
Es obligación humana darle al individuo lo que es "suyo", lo que le corresponde: he aquí el fundamento en el que se basa toda justa ordenación sobre la tierra. Toda injusticia significa, en cambio, que le es retenido o quitado al hombre lo que es suyo, y que le es quitado o retenido no por desgracia, la mala cosecha, el fuego o el terremoto, sino por la acción humana individual o institucional.

En un mundo globalizado, donde abundan los conflictos, el valor justicia redimensiona su sentido a fin de contrarrestar la consecuencia de la discriminación, la explotación y el abuso que se genera por las relaciones que se entretejen. Todos los seres humanos tienen un derecho natural para habitar el planeta y, lo contrario, sería injusto.

Macintyre (1994) expresa que el término justicia está ligado a la idea de acuerdo, a lo útil o equilibrado en las relaciones humanas, a lo que es bueno, al reconocimiento de los derechos humanos. También se asemeja al orden o a la medida. Se dice que hay justicia cuando cada cosa ocupa su lugar.

La justicia como valor, dirige la acción humana al reconocimiento de los derechos de los demás y es un criterio que, como seres sociales nos insta a dar a cada quien lo que, por derecho, se merece.

\section{La paz}

La paz es el cimiento básico sobre el que descansa la construcción de las sociedades civilizadas y a partir del cual puede desarrollarse la convivencia. Señala Siliceo (1997, p.41) que "La paz social no es sólo un valor, un anhelo de todo hombre, grupo, empresa, nación y comunidad de naciones. La paz en un fenómeno sociopolítico, que ahora más que nunca es un requisito para la supervivencia del género humano".

Explican Ortega y Mínguez (2001) que el concepto de paz está indisolublemente ligado a la libertad del ser humano y a los derechos que realmente ejerce, pues si no hay libertad no existen acciones a favor de la paz. También señalan las siguientes características:

- La paz es obra de la justicia.

- La paz no es ausencia de guerra o violencia.

- La paz es un proceso, no el fin de un camino.

- La paz es fraternidad y exige solidaridad. 
- La paz exige el reconocimiento de la primacía y dignidad irrenunciables del ser humano.

En el contexto actual, la paz es verdaderamente uno de los compromisos más grandes que deben asumir los líderes, quienes han de luchar por ella, propiciarla, mantenerla y más aún, defenderla. Sin embargo, no es solo responsabilidad de los líderes presentes en cualquier contexto, sino también un compromiso del ciudadano y la ciudadana común.

Cuando hay diálogo, tolerancia, libertad, solidaridad, justicia y paz, como valores que guían al ser humano, la convivencia gana espacio en la diversidad social. Luego en estos valores se fundamenta el éxito en la práctica de la ética intercultural.

\section{La multiculturalidad y sus modelos}

El avance de la civilización y más en la actualidad, el fenómeno de la globalización, han propiciado la formación de sociedades multiculturales. Algunos ven la multiculturalidad como una consecuencia natural de la evolución y reconocen las ventajas que brinda la existencia de diferentes culturas, otros argumentan que:

la multiculturalidad se inserta en un modelo de desarrollo socioeconómico que lejos de llevarnos a un clima de igualdad y de justicia, ha profundizado las desigualdades existentes con todas sus secuelas; la violencia, el racismo, el sexismo, el consumismo, la ideología del éxito personal, el individualismo, la insolidaridad. (Antón y otros, citados por Millán, 2001, p. 3)

Por su parte, Millán (2001) sostiene que la multiculturalidad se reconceptualiza al considerarse que las sociedades heterogéneas y diversificadas tienen un gran potencial para la innovación, creatividad y apertura a los cambios sociales.

García y Granados (1999, citados por Millán, 2001) ilustran diferentes modelos de educación multicultural que permiten abordar la multiculturalidad.

1. Educar para igualar: la asimilación cultural. Este modelo intenta igualar las oportunidades educativas entre grupos minoritarios o marginados y grupos favorecidos, procurando que los sujetos diferentes sean competitivos en la cultura dominante. 
2. El entendimiento cultural: el conocimiento de la diferencia. Preparar al estudiante para vivir en armonía en una sociedad multiétnica, orientarlos hacia el enriquecimiento cultural.

3. El pluralismo cultural: preservar y entender el pluralismo. Este modelo ve las diferencias como valiosas y necesarias para el bienestar de la sociedad, lo que expresa un pluralismo cultural que requiere de cuatro condiciones:

- Existencia de diversidad cultural dentro de la sociedad

- Interacción inter e intragrupos

- Los grupos que coexisten deben compartir las mismas oportunidades políticas, económicas y educativas.

- La sociedad debe valorar la diversidad cultural.

El pluralismo debe sustituir al etnocentrismo y afrontar la diversidad cultural en y desde la educación.

4. La educación bicultural: la competencia en dos culturas. Este modelo busca la preparación de los individuos para ser competentes en dos culturas. La educación bicultural debe posibilitar la participación de los alumnos y las alumnas, bien del grupo mayoritario o del minoritario, en las oportunidades socioeconómicas, sin perder sus identidades.

5. La educación como transformación: educación multicultural y reconstrucción social. El modelo busca la concientización de los estudiantes y las estudiantes de los grupos minoritarios sobre sus orígenes, comunidad, padres, nivel socioeconómico, etc., para que sean capaces de actuar socialmente basados en la comprensión crítica de la realidad.

Según Millán (2001), estos enfoques muestran los diferentes tratamientos que la educación da a la multiculturalidad. Sin embargo, en la actualidad debe haber un cambio hacia el acercamiento intercultural, dejando de lado prácticas homogeneizadoras tradicionales. El cambio debe ser hacia la valoración de la diversidad: fuente de riqueza en el aula y premisa social para vivir en armonía.

Para Jordán y col (2002), la educación multicultural es el intento de adaptación de los sistemas de enseñanza a las necesidades de los diferentes grupos sociales. Señalan los autores que el multiculturalismo concede una valoración excesiva a las variables de la cultura de origen en la construcción de las identidades culturales a costa de la disminución de la capacidad del individuo para construir su propia identidad. Además, argumentan que la 
educación multicultural reconoce las diferencias pero las equipara, al parecer, con deficiencias, implementando esquemas pedagógicos de compensación que buscan equilibrar la situación; el problema es que, sin pretenderlo, se fomentan relaciones no igualitarias teñidas de paternalismo.

Al reconocer estas dificultades de la multiculturalidad, va cobrando mayor valor la interculturalidad, pues gana terreno como un modelo de gestión que administra con eficacia y eficiencia (con calidad) la diversidad cultural.

\section{Conclusiones}

El desarrollo de este ensayo ha permitido arribar a las siguientes conclusiones:

- La ética intercultural permite minimizar los riesgos del proceso globalizador, a través de la creación de espacios para el diálogo y la inclusión.

- La ética intercultural es una propuesta de acción que busca construir y reconstruir sistemas de valores que aseguren la convivencia social.

- La educación debe asumir su papel activo como responsable de la implementación de la ética intercultural; por un lado a través de una formación en valores que desarrolla, propicia y respeta los mínimos necesarios para convivir, y por otro, con una formación en la interculturalidad que se enriquece en la diversidad y concientiza respecto al reconocimiento del otro. Se justifica el planteamiento de una educación intercultural en el entendido de que las sociedades se han transformado en multiculturales y se requiere posibilitar la interacción entre sus miembros.

- La ética intercultural necesita de diálogo, tolerancia, libertad, solidaridad, justicia y paz para practicarse con éxito en la diversidad cultural que administra.

- La interculturalidad es un modelo de gestión que administra positivamente la diversidad cultural y que por ello va más allá de la multiculturalidad, ya que no basta con el reconocimiento de las diferencias, se requiere un enfoque mucho más comprometido, que ponga frente a frente esas diferencias y que, a través de la participación, la responsabilidad, el diálogo, la tolerancia y la lucha contra la injusticia, posibilite la existencia de un espacio común para la vida en armonía entre seres diversos.

Finalmente, es importante resaltar que la práctica de la ética intercultural se posibilita a través de una educación intercultural que educa para el respeto a la diversidad y que al buscar la convivencia entre diferentes, promueve la construcción y reconstrucción de valores, 
por lo tanto educa en valores. Concretando, la educación intercultural se fundamenta en una educación en valores, al reconocer que el diálogo, la tolerancia, la libertad, la solidaridad, la justicia y la paz deben arraigarse y desarrollarse en un ser humano que se prepara para la vida en sociedades culturalmente diversas; todo ello contribuye al fortalecimiento de la ética intercultural, y dando respuesta a la inquietud inicial de este ensayo, permitirá alcanzar la convivencia, enfrentando los riegos que implica el proceso de globalización.

\section{Referencias}

Antón, José; Lluch, Xavier y Rodas, María. (1995). Educar desde el interculturalismo. Salamanca, España: Amarú

Arana, Martha y Batista, Nuris. (2000). La educación en valores: una propuesta pedagógica para la formación profesional. Recuperado el 17 de febrero de 2001, de http://www.campus-oei.org/cts/ispajae.htm

Arrieta, Josetxu; Fernández, José y Alvarez, Jesús. (1995). El espacio de la educación para el desarrollo en el marco de la LOGSE. Recuperado el 7 de diciembre de 2004, de http://www.fuhem.es/CIP/EDUCA/art1hego.htm

Asociación de Enseñantes con Gitanos. (1996). Seminario de educación intercultural. Recuperado el 10 de noviembre de 2004, de http://www.pangea.org/aecgit/educacion.htm.

Comisión Pedagógica de S.O.S. Racismo Madrid. (1997). Propuestas para una educación intercultural. Recuperado el 10 de Noviembre de 2004, de http://www.cnice.mecd.es /interculturanet/archivos/propuestas.rtf

Dalla Costa, John. (1999). El imperativo ético. Por qué el liderazgo moral es un buen negocio. Barcelona, España: Paidos

Denis, Lourdes. (2000). Ética y docencia. Caracas, Venezuela: Fedupel

Diaz, Ricardo. (2005). La bella ilusión de la tolerancia. Recuperado el 15 de febrero de 2008, de http://www.ricardodiaz.org/archives/2005/10/la bella ilusio.html

García, Joaquín. (1994). Solidaridad y voluntariado. Santander: Sal Térrea

García, Javier y Granados, Antolín. (1999). Lecturas para educación intercultural. Madrid: Trotta

Gómez, María, Miró, Pau, Lozano, Félix, Boni, Alejandra, Ciurana, Juan y Calabuig, Carola. (2004). Apuesta por la transversalidad de los valores éticos en la docencia universitaria en los estudios científico-técnicos. Recuperado el 17 de enero de 2008, de http://www.grend.webs.upv.es/presentacion files/recursos/Apuesta\%20por \%20la\%20 transversalidad\%20Cuba\%202004.pdf 
Guédez, Víctor. (2001). La ética gerencial. Instrumentos estratégicos que facilitan decisiones correctas. Caracas, Venezuela: Fedupel

Ferrer, Juliana y Romero, Douglas. (2003). Educando una nueva ética global. Revista de Estudios Interdisciplinarios en Ciencias Sociales Telos, 5 (3): 327-342.

Flimus, Daniel. (1998). Introducción. En Las Transformaciones educativas en Iberoamérica. Tres desafíos. Democracia, desarrollo e integración. Argentina: Troquel

Fornet-Betancourt, Raúl. (2001). Transformación intercultural de la filosofía. Bilbao, España: Brouwer

Hoyos, Guillermo. (1998). Educación y ética para ciudadanos. En Daniel Flimus (Comp.), Las Transformaciones educativas en Iberoamérica. Tres desafíos. Democracia, desarrollo e integración. Argentina: Troquel.

Huntington, Samuel. (1996). The clash of civilizations and the remaking of World order. Nueva York: Simón \& Schuster.

Jordán, José; Ortega, Pedro y Minguéz, Ramón. (2002). Inmigración: una respuesta desde la pedagogía intercultural (Ponencia) XXI Seminario interuniversitario de Teoría de la Educación. Recuperado el 20 de noviembre de 2007, de http://www.ucm.es/info/site /docu/21site/a2escamez.pdf

Kant, Immanuel. (1994). Hacia la paz perpetua. Madrid: Biblioteca Nueva

Macintyre, Alasdair. (1994). Justicia y racionalidad. Barcelona, España: Ediciones Internacionales Universitarias.

Martínez, Vicent. (2000). Cultura y paces: educar para la interculturalidad. Recuperado el 7 de diciembre de 2005, de http://www.ua-ambit.org/jornadas2000/Ponencias/j00vicent-martinez.htm.

Millán, María. (2001). Interculturalidad y educación. Recuperado el 25 de noviembre de 2001, de http://redderedes.upn.mx/2areunion/lupemillan.htm.

Ortega, Pedro y Mínguez, Ramón. (2001). Los valores en la educación. Barcelona, España: Ariel Educación.

Petty, Miguel. (1998). ¿Quién se ocupa de la educación de valores? En Las Transformaciones educativas en Iberoamérica. Tres desafíos. Democracia, desarrollo e integración. Flimus, Daniel. Compilador. Argentina: Troquel.

Ramos, María. (2000). Programa para educar en valores. La educación que transformará al país. El Hatillo, Venezuela: Ediciones Paulinas.

(2001). Para educar en valores. Teoría y práctica. El Hatillo, Venezuela: Ediciones Paulinas. 
Salas, Ricardo. (2002). Problemas y perspectivas de una ética intercultural en el marco de la globalización cultural. Revista de Filosofía, 2 (41): 7-29.

Sarasola, José. (2000). Solidaridad y voluntariado: una visión crítica. Recuperado el 20 de marzo de 2001, de http://www.redalyc.uaemex.mx/redalyc/pdf/158/15801515.pdf

Siliceo, Alfonso. (1997). Líderes para el siglo XXI. México: Mc Graw Hill.

Toro, Alfredo. (1998). La globalización es un hecho, no una ideología. Caracas, Venezuela: El Universal. 